\title{
Exploitation of multilayer coatings for infrared surface plasmon resonance fiber sensors
}

\author{
T. Allsop, ${ }^{1, \star}$ R. Neal, ${ }^{2}$ C. Mou, ${ }^{1}$ P. Brown, ${ }^{2}$ S. Saied,${ }^{3}$ S. Rehman, ${ }^{1}$ K. Kalli, ${ }^{4}$ D. J. Webb,${ }^{1}$ \\ J. Sullivan, ${ }^{3} \mathrm{D}$. Mapps, ${ }^{2}$ and I. Bennion ${ }^{1}$ \\ ${ }^{1}$ Photonics Research Group, Aston University, Aston Triangle, Birmingham, B4 7ET, UK \\ ${ }^{2}$ Department of Communications and Electrical Engineering, Faculty of Technology, \\ University of Plymouth, Plymouth, PL4 8AA, U.K \\ ${ }^{3}$ Surface Science Research Group, Aston University, Aston Triangle, Birmingham, B4 7ET, UK \\ ${ }^{4}$ Cyprus University of Technology, Department of Electrical Engineering and Information Technology, \\ 31 Archbishop Kyprianos, Lemessos 3036, Cyprus \\ ${ }^{*}$ Corresponding author: t.d.p.allsop@aston.ac.uk
}

Received 12 August 2008; revised 17 November 2008; accepted 25 November 2008; posted 2 December 2008 (Doc. ID 100058); published 7 January 2009

\begin{abstract}
We demonstrate surface plasmon resonance (SPR) fiber devices based upon ultraviolet inscription of a grating-type structure into both single-layered and multilayered thin films deposited on the flat side of a lapped D-shaped fiber. The single-layered devices were fabricated from germanium, while the multilayered ones comprised layers of germanium, silica, and silver. Some of the devices operated in air with high coupling efficiency in excess of $40 \mathrm{~dB}$ and an estimated index sensitivity of $\Delta \lambda / \Delta n=90 \mathrm{~nm}$ from 1 to 1.15 index range, while others provided an index sensitivity of $\Delta \lambda / \Delta n=6790 \mathrm{~nm}$ for refractive indices from 1.33 to 1.37 . (C) 2009 Optical Society of America

OCIS codes: $\quad 060.2370,060.2310$.
\end{abstract}

\section{Introduction}

Gratings in fibers-including long period gratings (LPGs), fiber Bragg gratings, and tilted fiber Bragg gratings - and surface plasmon resonance (SPR) (in both planar and fiber configurations) may all be used to detect changes in the refractive index of various substances with potential applications in chemistry, biochemistry, and biology [1-5]. SPR is an important optical phenomenon that involves a resonant transfer of the incident light energy to a surface plasmon (SP) mode in the form of collective electron oscillations in a metal [6]. It has been found that SP generation is very sensitive to the polarization of the illuminating light, its wavelength, and its angle of incidence on the metal surface. This sensitivity can be used to detect index changes in biochemical/che-

0003-6935/09/020276-11\$15.00/0

(C) 2009 Optical Society of America mical reactions [7]. SPR biosensors offer the opportunity for real-time and label-free monitoring of biomolecular interactions [8].

The plasmons exist at a metal-dielectric interface and obey the following dispersion relation for two homogeneous semi-infinite media [7]:

$$
\beta=k \sqrt{\left(\frac{\varepsilon_{m} \cdot n_{s}^{2}}{\varepsilon_{m}+n_{s}^{2}}\right)},
$$

where $k$ is the free space wavenumber, $\varepsilon_{m}$ is the dielectric constant of the metal $\left(\varepsilon_{m}=\varepsilon_{m r}+i \varepsilon_{m i}\right)$, and $n_{s}$ is the refractive index of the dielectric.

There is a considerable body of published research relating to fiber-grating-based refractive index sensors with the majority of these devices. The highest index sensitivity is obtained with test sample indices over $\sim 1.4$ [9], and they generally have less sensitivity in the aqueous index regime. This problem is being 
addressed with the use of tilted Bragg gratings to assist in the generation of SPRs in a fiber configuration $[10,11]$. There is very little published with regards to multilayered thin film SPR fiber devices, and the majority of these papers, such as $[12,13]$, address the SP's optical properties and not their sensing potential.

We report on a novel SPR fiber device based upon a surface relief grating-type structure inscribed with UV light into a multilayered thin film deposited on the flat side of a lapped, D-shaped fiber. Unusually, this device exhibits coupling to the SP when surrounded by air.

\section{Fabrication and Characterization}

The fiber devices were constructed in three stages [10]. First, a standard single mode fiber was mechanically lapped down to $10 \mu \mathrm{m}$ from the corecladding interface. Second, using RF sputtering, a series of coatings were deposited upon the flat of the lapped fiber. These coatings consisted of various layers of germanium, silicon dioxide, and silver, as described below. Third, the coated fiber was exposed to a UV light interference pattern produced by a uniform phase mask with period $1.018 \mu \mathrm{m}$ through laser beam scanning and multiexposure. This produced a surface relief structure that has dominant spatial periods of $\sim 0.5 \mu \mathrm{m}$ and $\sim 1 \mu \mathrm{m}$.

The coatings used were a single coating of germanium of average thickness $48 \mathrm{~nm}$, a two-layered coating of germanium $(48 \mathrm{~nm})$ followed by silicon dioxide $(48 \mathrm{~nm})$, and a three-layered coating of germanium $(48 \mathrm{~nm})$ followed by silicon dioxide $(48 \mathrm{~nm})$ followed by silver $(32 \mathrm{~nm})$. The rationale for using these materials is in two parts. The first concerns the optical constants of the materials and how their dispersion relationships allow coupling to surface plasmons at a metal-dielectric or semiconductor-dielectric interface; both Ge and Ag exhibit this behavior. Second, germanium and and silicon dioxide layers are used due to the fact that it is known from studies of grating formation [14] that when exposed to UV light, $\mathrm{Ge} / \mathrm{GeO}$ produces photobleaching and compaction of the material, thus producing a surface corrugation on the multilayered structure. We believe that this compaction creates a periodic strain field in the coating and its fiber substrate extending into the core, which then acts to couple light from the core into the SP mode [15]. Also it is known that exposing germanium to an inhomogeneous energy deposition (such as a UV light interference pattern produced by a uniform phase mask) causes local melting of the surface, which then does not recrystallize flat and leaves a permanent and localized structure formed in the germanium and at higher laser fluences $\left(\sim 100 \mathrm{~mJ} / \mathrm{cm}^{2}\right.$ upwards) can cause a periodic structure $[16,17]$.

The fiber devices were investigated using a tunable laser system to measure the characteristic transmission spectra, the optical reflection spectra and polarization-dependent loss (DWDM Passive Component Test System-IQS-12004B from EXFO). As may be seen in Fig. 1, a broad spectral feature in the reflected spectrum was found (from $1530 \mathrm{~nm}$ to $1600 \mathrm{~nm}$ ), suggesting that there is some quasiperiodic index variation experienced by the core mode causing coupling to the counterpropagating core mode. This reflected feature is spectrally broad and weak, suggesting that the period of the index variation is varying along the core. A contribution to the spectral broadness may arise from strain variations brought about by spatial variation in the coating thickness or composition along the fiber. It was also found that the spectral features are polarization dependent, suggesting that the index perturbation across the core is not radially symmetric, which is to be expected for this kind of gratinglike structure. We also observed a narrow spectral feature at $1565 \mathrm{~nm}$ in transmission, in reflection (due to residual reflection at the fiber end), and in the polarization-dependent loss. This feature is spectrally sensitive to changes in the refractive index of the surrounding medium, and we believe it arises from coupling to a SP mode of the coating. The mechanism that promotes the surface relief modulation that occurs in the coating on UV light exposure and the subsequent coupling mechanism to the SP is currently
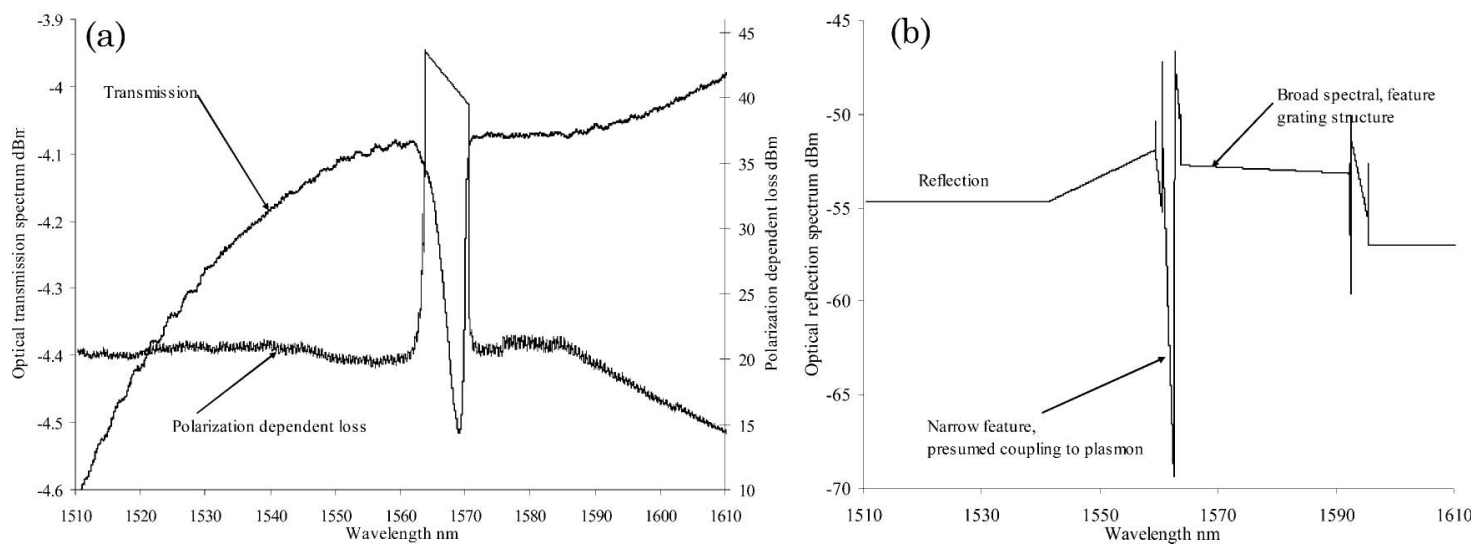

Fig. 1. Transmission and reflection spectra of post-UV exposed D-shaped fiber with a multilayered coating consisting of three layers, Ge-SiO ${ }_{2}$-Ag. (a) Polarization-dependent loss and transmission spectrum, (b) reflection spectrum and coupling to a SP. 
under investigation and will not be dealt with in this paper.

Prior to UV processing the polarization dependence had been investigated, and a small variation in the overall transmitted optical power with polarization was found. After UV exposure the devices were further characterized by observing the spectrum of the transmitted light as the azimuth of the polarization state was changed. To do this, light from a broadband light source was passed through a polarizer and a polarization controller before illumination of the sample, with the transmission spectra being monitored using an optical spectrum analyzer (accuracy of $0.005 \mathrm{~nm}$ ) the change in polarization of the illuminating light is monitored with a polarimeter (Tektronix, PAT 9000B) via a polarization maintaining coupler; see Fig. 2.

A broadband light source was used to illuminate each fiber device, and the transmission spectra were monitored during UV processing. Prior to the UV exposure it was found that the transmission spectra showed very little dependence on the polarization of the illuminating light, while during the UV processing, the transmission spectra showed a significant variation with polarization.

For the single coating of germanium, with air as the surrounding medium no coupling (stopband) was observed in the transmission spectrum, but at a refractive index of 1.3 and above a polarizationdependent attenuation band (resonance) was observed. With the two-layered coating, the transmission spectrum of the device changed significantly with UV exposure and was also polarization dependent. This time, when air was the surrounding medium, stopbands indicating coupling were observed in the transmission spectrum at two spectral location, $1316 \mathrm{~nm}$ and $1450 \mathrm{~nm}$, depending on the polarization of the incident light; see Fig. 3.

In the case of the three-layered device, during UV processing the transmission spectrum of the device changed significantly with UV exposure and appeared to have high polarization dependence. As in the case of the two-layered device, with air as the surrounding medium two stopbands were observed in the transmission spectrum for different polarization states, this time at $1370 \mathrm{~nm}$ and $1550 \mathrm{~nm}$ : see Fig. 4.

Due to the fact that no coupling appeared for the germanium-coated fiber device in air, its polarization dependence was investigated with a surrounding medium with refractive index 1.36, the response being typical for other similar indices. Only one stopband was present in the observed spectrum (1250$1650 \mathrm{~nm}$ ), though the stopband could be tuned over a spectral range of $70 \mathrm{~nm}$ by adjusting the polarization of the incident light. During the tuning, the peak strength of the spectral feature varied from $38 \mathrm{~dB}$ to $3 \mathrm{~dB}$; see Fig. 5(a) for the transmission spectra. Figures $5(\mathrm{~b})$ and $\overline{5(\mathrm{c})}$ show the evolution of the centroid wavelength and maximum coupling strength of the fiber device.

The two-layered coating was investigated for polarization dependence; Fig. 6(a) shows the change in the transmission spectra.

Investigating the polarization dependence of the Ge-SiO ${ }_{2}$-coated fiber device, it can be seen from Fig. $\underline{6}$ that these two spectral transmission features appear to respond to polarization in opposite ways; see Fig. 6(b). The wavelength shift of the shorter wavelength resonance has greater sensitivity to the polarization of the illuminating light than the higher one.

Inspecting the polarization properties of the $\mathrm{Ge}$ $\mathrm{SiO}_{2}$-Ag-coated fiber device, it was found that it had a higher sensitivity to polarization than that coated with $\mathrm{Ge}^{-\mathrm{SiO}_{2}}$; see Figs. 6(c) and 7(c). Also a higher sensitivity to polarization of the $\mathrm{Ge}^{-\mathrm{SiO}_{2}}$ $\mathrm{Ag}$ device was found for the shorter wavelength $(1390 \mathrm{~nm})$ band. Again, the two resonances seem to respond to polarization in opposite fashions; see Fig. 7(b).

We are mostly concerned with the potential of these SPR devices for chemical and biochemical sensing applications using wavelength and intensity interrogation. While it may seem that the polarization sensitivity of these SPR devices may be problematic,

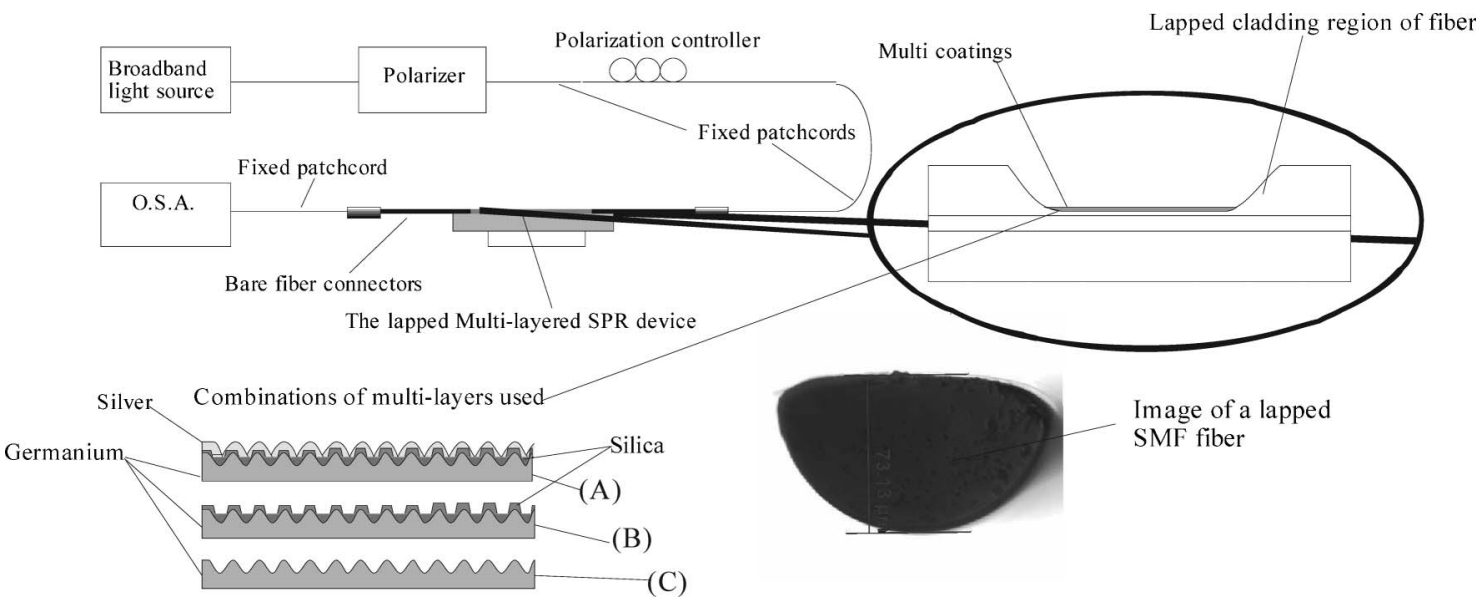

Fig. 2. Scheme used for the characterization of the lapped and multilayer-coated fiber devices and the combinations of layers used in the

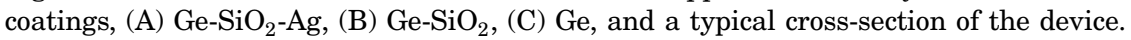




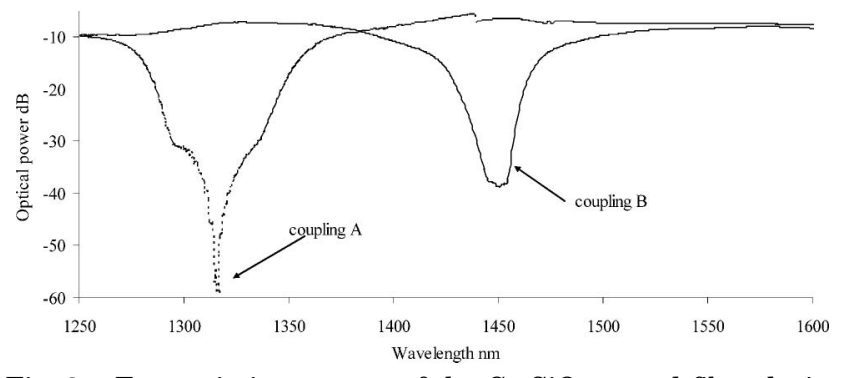

Fig. 3. Transmission spectra of the $\mathrm{Ge}_{-} \mathrm{SiO}_{2}$-coated fiber device surrounded by air for two polarization states of the illuminating light.

this can potentially be overcome by using polarization maintaining fiber [3], or can be seen as another spectral property to be exploited. A detection scheme that does exploit this behavior is angular interrogation [18]. Inspecting the transmission spectra in Figs. $\overline{3}-\underline{7}$, there is no significant observed spectral feature associated with Bragg reflection from the gratings themselves. This is expected due to the fact their transmission profiles are very weak and that the interrogating light source is broadband and swamps the response shown in Fig. 1 .

\section{Refractive Index Sensitivity}

For refractive index sensitivity measurements the devices were placed in a V-groove and immersed in certified refractive index liquids (supplied by Cargille Laboratories Inc.), which have a quoted accuracy of \pm 0.0002 . The devices and V-groove were carefully cleaned, washed in ethanol, then in deionized water, and finally dried before immersion into the next certified refractive index liquid. The Vgroove was made in an aluminum plate, machined flat to minimize bending of the fiber. The plate was placed on an optical table, which acted as a heat sink to maintain a constant temperature. The V-groove was used in conjunction with the apparatus shown

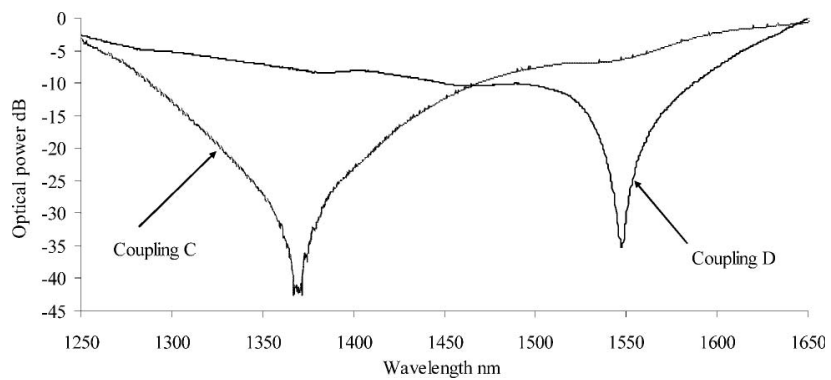

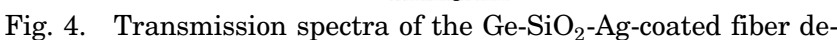
vice surrounded by air for two polarization states of the illuminating light.

in Fig. 2. Figures $8-11$ show the spectral sensitivity of the three devices to refractive index.

Inspecting Fig. 8 shows that at higher surrounding index "ripples" start to appear in the transmission profile of the germanium-coated device, which may cause problems in measuring the wavelength shift without using a centroid method, which is the approach used to obtain the data in Fig. 8(b). Changing the polarization state of the illuminating light decreased these spectral "ripples" observed with the germanium-coated SPR devices. The "ripples" were not observed with any of the other devices.

Inspecting the spectral characteristics of the $\mathrm{Ge}^{-\mathrm{SiO}_{2}}$-coated fiber device as a function of index (Figs. 9(b) and 9(c)), it can be seen that the two resonances display distinct behavior, with the shorter wavelength feature having the higher spectral and coupling sensitivity to changes in the surrounding refractive index. On the other hand, for the $\mathrm{Ge}_{-} \mathrm{SiO}_{2}-\mathrm{Ag}$ device, the longer wavelength feature has the higher spectral and coupling sensitivity to the surrounding index. This illustrates the dramatic change in spectral behavior that occurs with the addition of a silver layer of $32 \mathrm{~nm}$ thickness.

Comparing the index sensitivities of these devices to other fiber devices (such as LPGs) in the aqueous
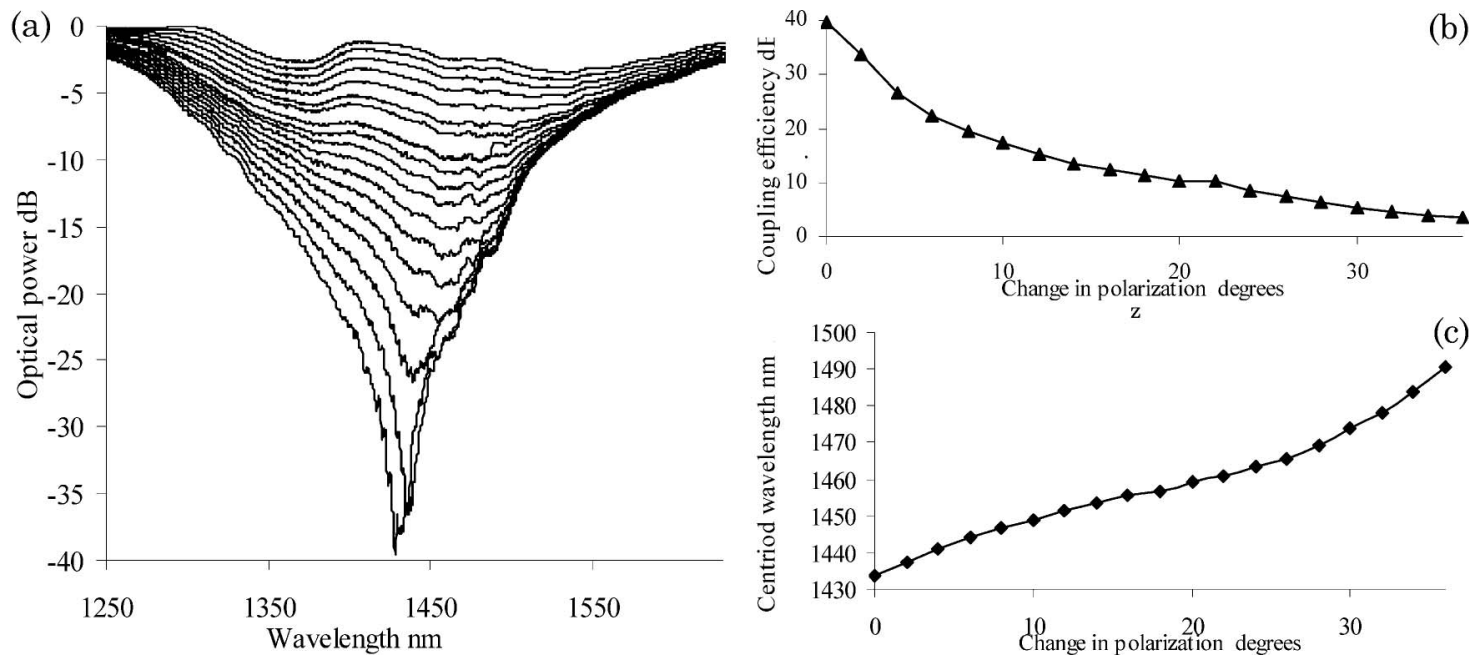

Fig. 5. (a) Transmission spectra of the Ge-coated fiber device surrounded by a medium with a refractive index of 1.36 with changing polarization. (b) Coupling efficiency and (c) wavelength shift of the resonance as the azimuth of polarization of the illuminating light is changed from the position providing maximum coupling. 

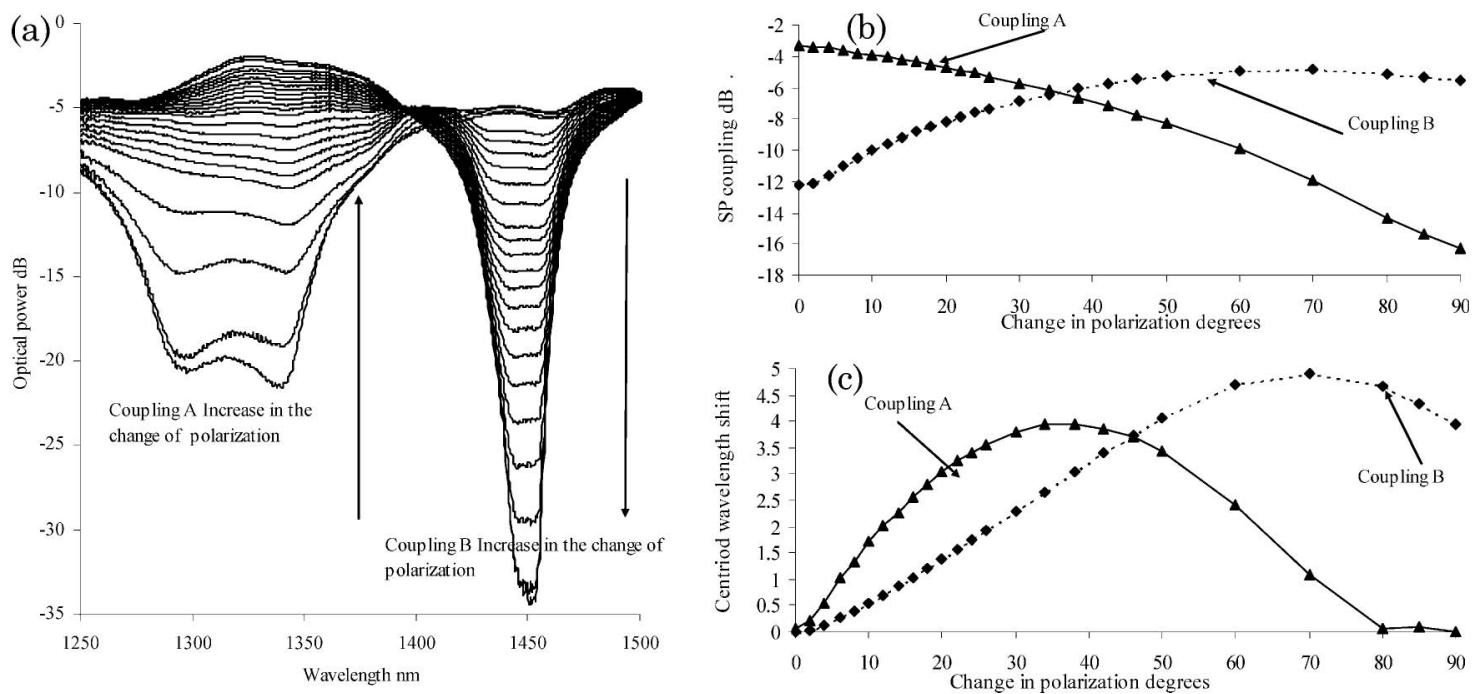

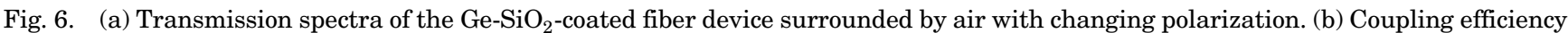
and (c) wavelength shift of the resonance as the azimuth of polarization of the illuminating light is changed from the position providing maximum coupling at $1450 \mathrm{~nm}$.

index regime (1.33 to 1.39 ) gave comparable or more favorable results. The highest wavelength spectral sensitivity to index was obtained with the germanium coating, which provided $\Delta \lambda / \Delta n$ ranging from $3200 \mathrm{~nm}$ to $12,500 \mathrm{~nm}$; see Fig. 12 . This estimate of index sensitivity is obtained by a linear regression technique to minimize the residuals of the wavelength shift as a function of the surrounding medium's index, yielding $\Delta \lambda \approx 0.002 n_{s}{ }^{44.0}$. The functional form was chosen empirically to provide a reasonable fit to aid sensitivity estimations and does not correspond to any theoretical description. This type of index sensing device yields a limiting index resolution of $5 \times 10^{-6}$ (assuming a spectral interrogation technique with a resolution of $0.1 \mathrm{~nm}$ ), which is one of the highest resolutions reported. The $\mathrm{Ge}_{-} \mathrm{SiO}_{2}-\mathrm{Ag}$ coated device produced sensitivities from $710 \mathrm{~nm}$ to $1200 \mathrm{~nm}$ in the aqueous regime, which is again favorable compared to other fiber devices; see Table $\underline{1}$.

\section{Discussion}

The most interesting response of these devices is the coupling when surrounded by air for both the $\mathrm{Ge}_{-} \mathrm{SiO}_{2}$ and $\mathrm{Ge}_{-} \mathrm{SiO}_{2}-\mathrm{Ag}$ coatings and the dramatic changes when the devices were submerged into an index around 1.3; see Figs. 9-11. This behavior is rather distinct from that of other optical fiber index sensors. It was found for the $\mathrm{Ge}_{-} \mathrm{SiO}_{2}-\mathrm{Ag}$ coating that
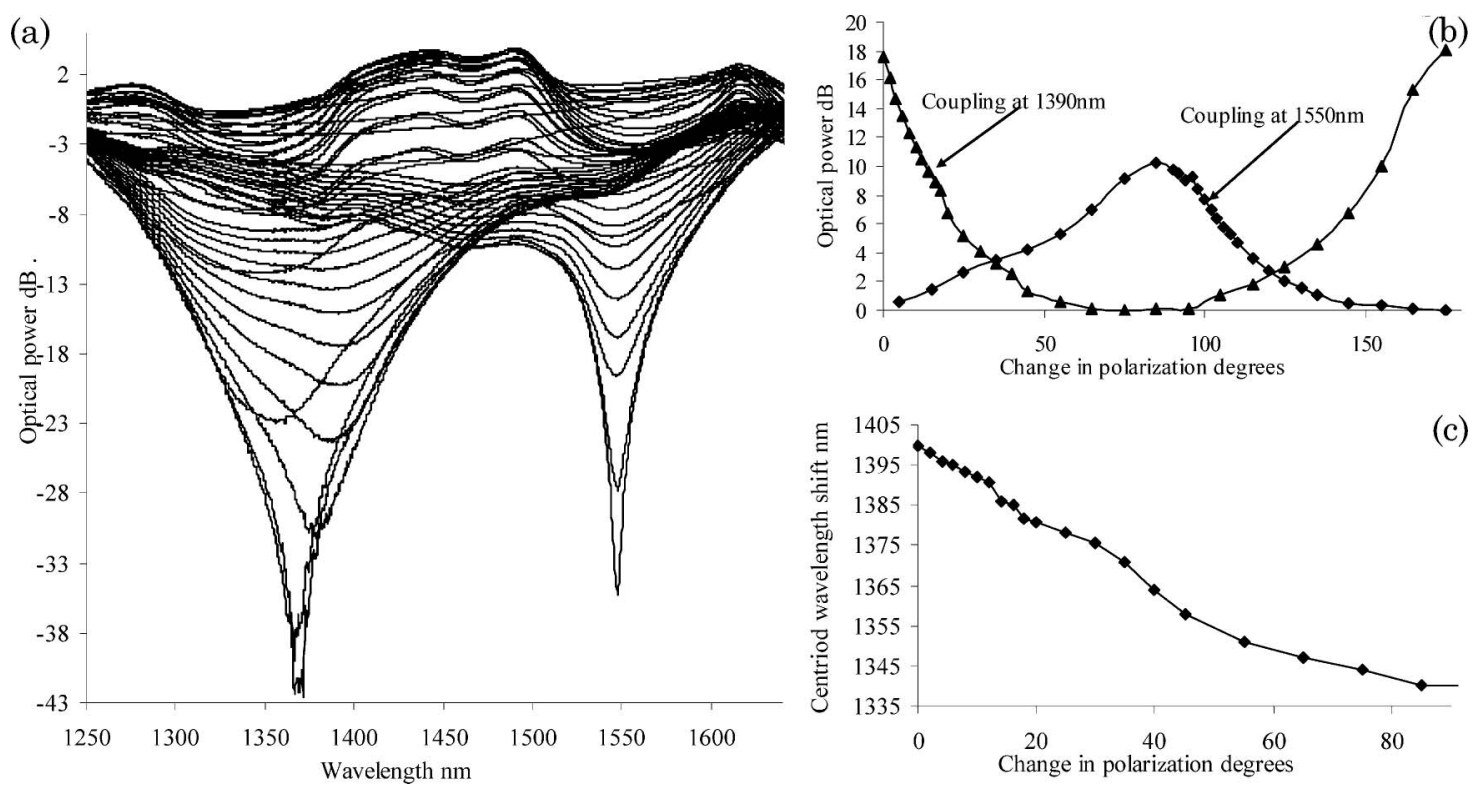

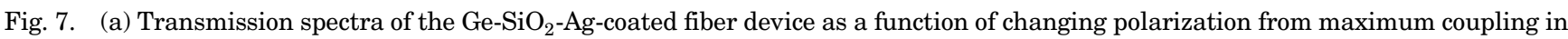
air. (b) Coupling efficiency of the resonances at $1390 \mathrm{~nm}$ and $1550 \mathrm{~nm}$ and (c) wavelength shift of the coupling feature at $1390 \mathrm{~nm}$ as the azimuth of polarization of the illuminating light is changed from the position providing maximum coupling. 

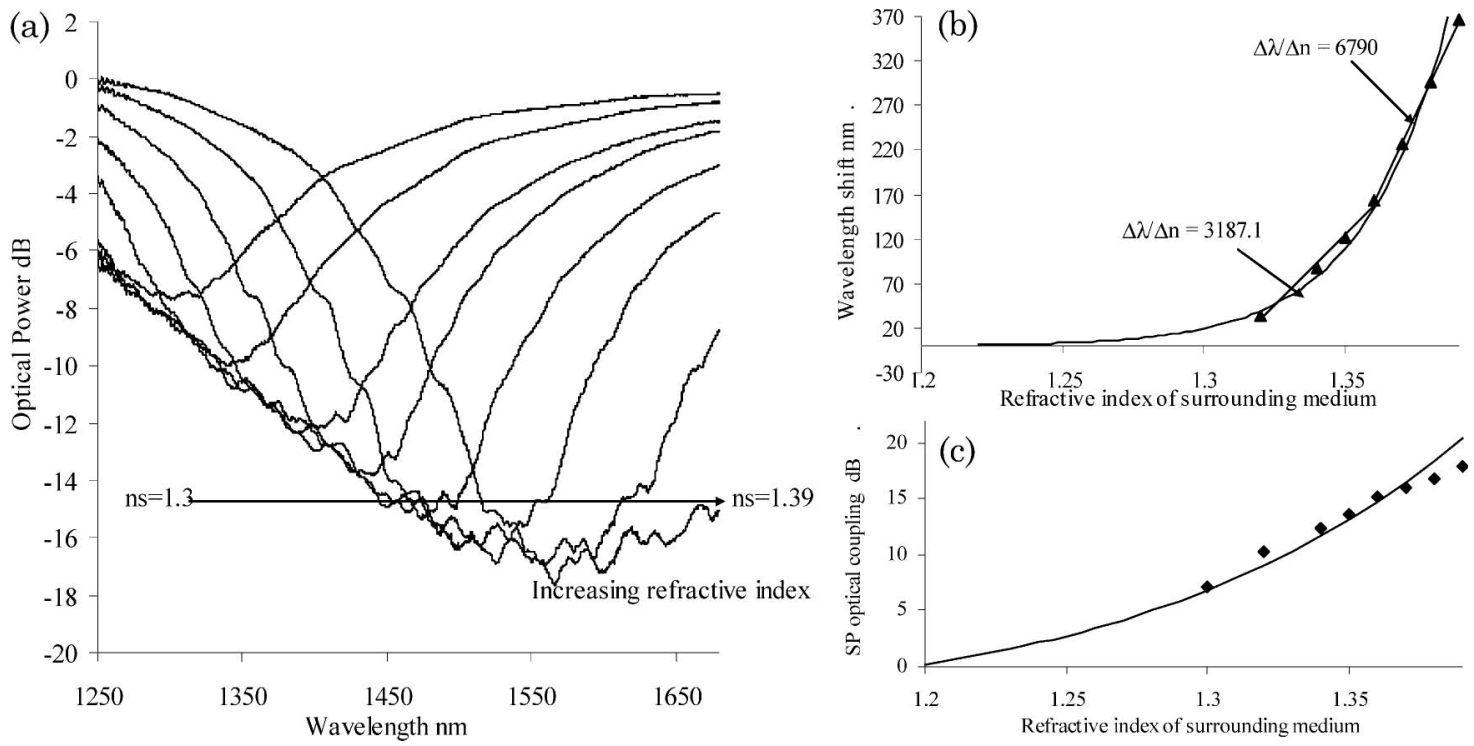

Fig. 8. (a) Transmission spectra of the germanium-coated device as a function of refractive index (polarization of the illuminating light chosen to maximize coupling at index 1.3). (b) Wavelength shift and (c) coupling strength of the resonance as a function of refractive index.

the wavelength shift as a function of surrounding index is approximately (and empirically) given by $\Delta \lambda=1.30 n_{s}{ }^{13.7}$, within the aqueous index regime. For the $\mathrm{Ge}_{-} \mathrm{SiO}_{2}$ coating, the wavelength shift as a function of surrounding index is $\Delta \lambda=0.33 n_{s}{ }^{14.4}$ within the aqueous index regime. To obtain an estimate of the spectral sensitivity of these SPR devices for low refractive indices from 1 to 1.1 we first use the same approach as in Ref. [11], which is to calculate the propagating modes of a D-shaped fiber with coating using a conformal mapping technique and with the germanium dispersion relationship obtained from Ref. [24]. It is assumed that light in the core is coupled to these $\mathrm{D}$-shaped fiber modes via the quasi-periodic strain field setup by the UV exposure. The next step is to implement Fresnel's equations for a three- or four-layered system (depending on coating structure being modeled) for different refractive
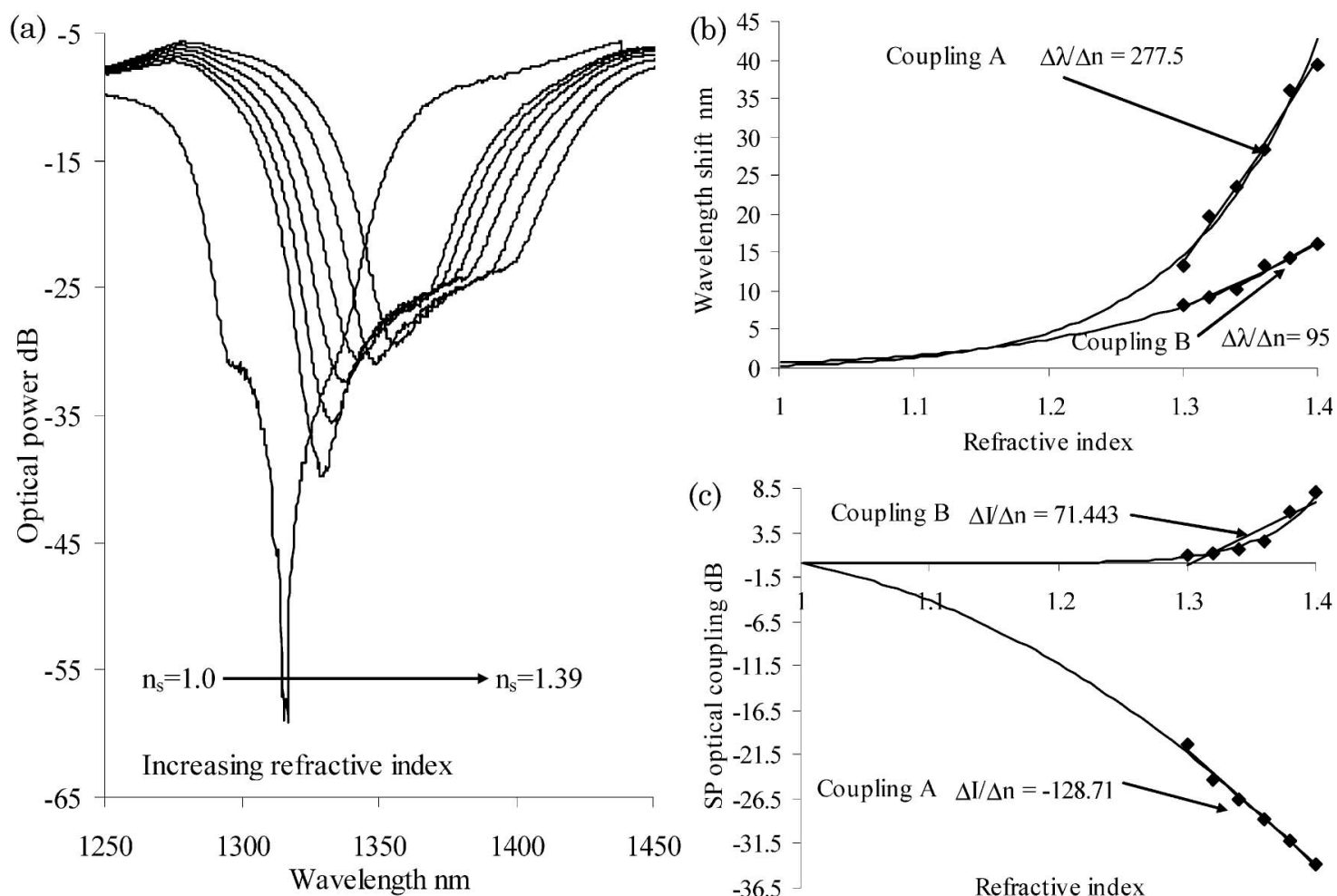

Fig. 9. (a) Transmission spectra of the $\mathrm{Ge}_{-} \mathrm{SiO}_{2}$-coated device as a function of refractive index (polarization of the illuminating light chosen to maximize coupling at index 1.3). (b) Wavelength shift and (c) coupling strength of the resonance as a function of refractive index. 
Table 1. Comparison of fiber Device Index Sensitivity in the Index Regime of 1.33 to 1.39

\begin{tabular}{|c|c|c|c|}
\hline Fiber Device & Index Sensitivity $\mathrm{d} \lambda / \mathrm{d} n_{s}$ & Index Resolution & Current Work \\
\hline Ge-coated SPR fiber device & $3200 \mathrm{~nm}$ to $12500 \mathrm{~nm}$ & $5 \times 10^{-5}$ to $5 \times 10^{-6}$ & \\
\hline Tilted FBG Ag coated SPR fiber device & $2100 \mathrm{~nm}$ to $3400 \mathrm{~nm}$ & $2 \times 10^{-5}$ to $5 \times 10^{-5}$ & [19], p. 5456. \\
\hline Step-index fiber tapered Mach-Zehnder & $643 \mathrm{~nm}$ to $713 \mathrm{~nm}$ & $5 \times 10^{-5}$ to $1 \times 10^{-4}$ & [21] p. 1702; [22]p. 471. \\
\hline FBG evanescent field & - & $2 \times 10^{-4}$ to $1 \times 10^{-3}$ & {$[2]$, pp. 757,764} \\
\hline Single mode fiber SPR sensor (fiber in a block) & $630 \mathrm{~nm}$ to $3300 \mathrm{~nm}$ & $3 \times 10^{-5}$ to $2 \times 10^{-4}$ & {$[2 \overline{3}]$ p. $74 ;[4]$, p. 3.} \\
\hline
\end{tabular}

indices of the surrounding medium, for example calculating the reflected intensity of the Ge coating on the D-shaped fiber for $p$-polarized light. The quantitative description of the minimum of the reflected intensity $R$ for a SPR is given by Ref. [7].

$$
R=\left|\frac{E_{r}^{p}}{E_{0}^{p}}\right|^{2}=\left|\frac{r_{n_{2} n_{\mathrm{sc}}}^{p}+r_{n_{\mathrm{sc}} n_{s}}^{p} \cdot \exp \left(2 i k_{\mathrm{zn}} d\right)}{1+r_{n_{2} n_{\mathrm{sc}}}^{p} \cdot r_{n_{\mathrm{sc}} n_{s}}^{p} \cdot \exp \left(2 i k_{\mathrm{zn}} d\right)}\right|^{2},
$$

where $E_{0}^{p}$ is the incident and $E_{r}^{p}$ is the reflected field, $d$ is the thickness of the germanium coating, and $r_{i, j}^{p}=\left(\frac{\mathrm{K}_{z, i}}{\varepsilon_{i}}-\frac{\mathrm{K}_{z, j}}{\varepsilon_{i}}\right) /\left(\frac{\mathrm{K}_{z, i}}{\varepsilon_{i}}+\frac{\mathrm{K}_{z, j}}{\varepsilon_{i}}\right)$ are the $P$-polarization amplitude reflection coefficients between layers $i$ and $j$, where $\mathrm{K}_{z, j}$ are the wave vector components of the incident light normal to each layer and $\varepsilon_{j}$ are the permittivities of the $j$ th layer, $n_{\mathrm{sc}}$ is the index of Ge, $n_{s}$ is the effective index of the surrounding medium, and $n_{2}$ is the index of the fiber cladding. The wave vector components $\left(\mathrm{K}_{z, j}\right)$ are determined from the calculated coated D-shaped fiber modes.

In evaluating the permittivity of the surrounding medium that affects the SP, the physical quality (uniformity, roughness) of the layers has to be considered within the calculations. Each layer has a significant surface roughness compared to its thickness, which would affect the overall optical constants of the materials in the layers. This roughness/thickness varia- tion was measured using an atomic force microscope (AFM) in conjunction with data analysis software NANORULE (Pacific Nanotechnology [25]), typically yielding an average thickness of $28 \mathrm{~nm}$. Also the measured average roughness of the silver coatings was $9 \mathrm{~nm}$ with the thickness ranging up to as much as $68 \mathrm{~nm}$, for an estimated sputtering thickness of $36 \mathrm{~nm}$. To estimate this effect on the optical constants we implemented an effective medium approximation: the Maxwell-Garnett theory [26], which yields an effective dielectric function as a function of fractional volume of the metal/semiconductor within an effective layer:

$$
\varepsilon_{\mathrm{eff}}=\frac{\left(3-2 V_{r}\right) \varepsilon_{i}+2 V_{r} \varepsilon_{h}}{V_{r} \varepsilon_{i}+\left(3-V_{r}\right) \varepsilon_{h}} \cdot \varepsilon_{h}
$$

where $\varepsilon_{\text {eff }}$ is the (complex) effective dielectric constant of the medium, $\varepsilon_{h}$ is the host material's dielectric function (for example for the $\mathrm{Ge}-\mathrm{SiO}_{2}$ coating, $\mathrm{SiO}_{2}$ is the host material), and $\varepsilon_{i}$ is the inclusion material (in this case the surrounding medium). The symbol $V_{r}$ represents the fractional volume of the host material within a volume. The total volume of coating and surrounding medium is based upon on the area of the coating multiplied by the spatial extension of the evanescent field perpendicular to the
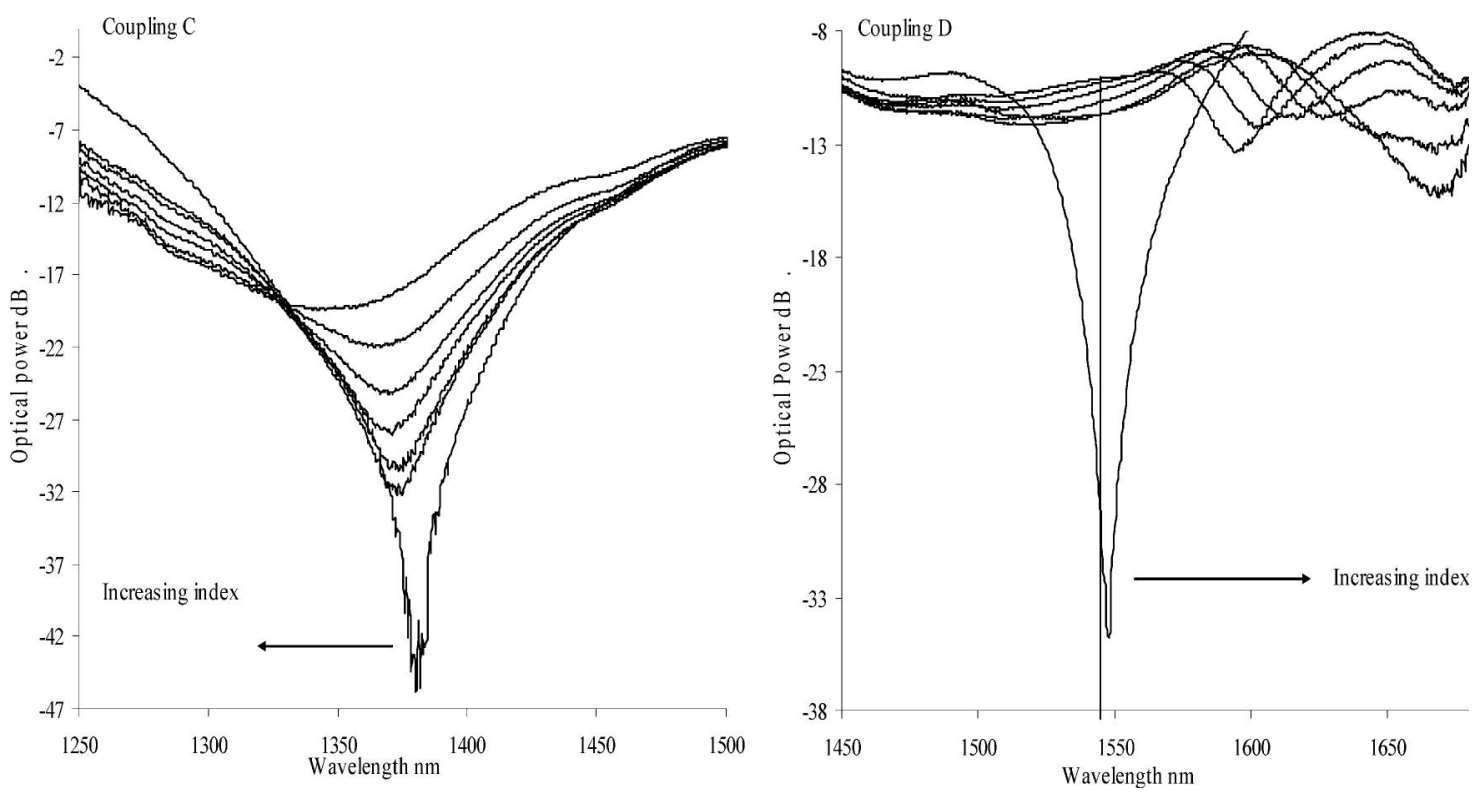

Fig. 10. Transmission spectra of the $\mathrm{Ge}_{-} \mathrm{SiO}_{2}$-Ag-coated fiber device as a function of refractive index. The polarization was adjusted to give maximum coupling at $1390 \mathrm{~nm}$ and $1550 \mathrm{~nm}$. 

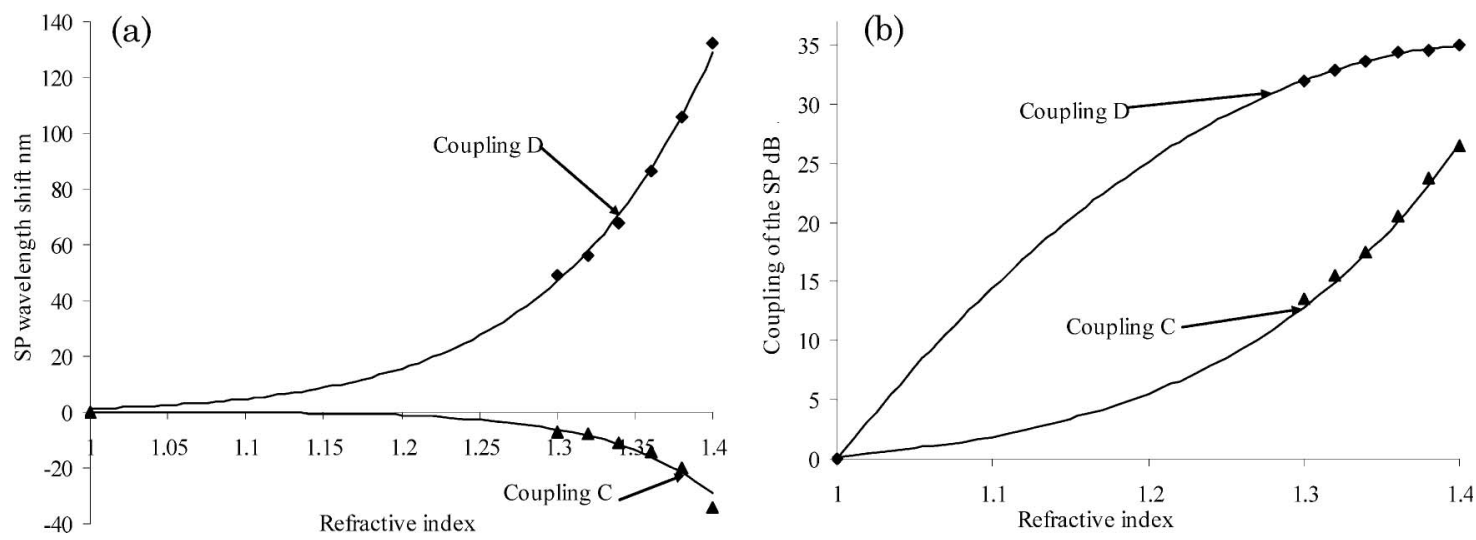

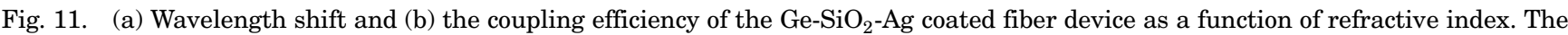
polarization was adjusted to give maximum coupling at $1390 \mathrm{~nm}$ (coupling C) and $1550 \mathrm{~nm}$ (coupling D).

coatings surface associated with the SP at a given wavelength and refractive index of the surrounding medium, thus giving a sensing volume. The spatial extension of the plasmon's evanescent field is calculated from the material constants of the two materials forming the metal-dielectric interface (the cladding of the fiber and the layer of $\mathrm{Ge}$ ) [27]:

$$
z_{\mathrm{sp}}=\operatorname{Im}\left[\frac{\sqrt{\left(\varepsilon_{\mathrm{Ge}}(\lambda)+n_{s}(\lambda)^{2}\right)} \cdot \lambda}{4 \pi n_{s}(\lambda)^{2}}\right]
$$

where $\varepsilon_{\mathrm{Ge}}(\lambda)$ is the dielectric constant of Ge and $n_{s}(\lambda)$ is the medium's refractive index as a function of wavelength. The volume of host material is estimated from the atomic force microscope measurements of the coatings described above. Therefore the effective dielectric constant is a direct function of the spatial extension of the evanescent field.

Using the approach outlined above for the Ge coating with the optical constants for germanium given in Ref. [28] we obtained reasonable results compared to the experimental data; see Fig. 12. We were unable to obtain such agreement using the data given in
Ref. [24], which raises questions as to the true values for the index and the extinction coefficient as a function of wavelength for germanium.

The results show a reasonably high index sensitivity of $\Delta \lambda / \Delta n=3274 \mathrm{~nm}$, but this is rather different from the figure obtained experimentally with the UV postfabrication of the coatings, which is understandable. There are several deficiencies in the simple model: the surface corrugation and depth variation have only been included simplistically, and no account has been taken of the fact that an inhomogeneous energy deposition can change the physical properties of the germanium $[\underline{16}, 17]$.

This modeling procedure was also implemented on a coating structure consisting of $\mathrm{Ge}_{-} \mathrm{SiO}_{2}$ and $\mathrm{Ge}-$ $\mathrm{SiO}_{2}-\mathrm{Ag}$, using material constants given by Ref. [24]. Again these results show similar spectral behavior to that observed experimentally; see Figs. 13 and 14 .

Comparing the theoretical data shown in Figs. 12 14 to the experimental data in Figs. 9-11, there is reasonable agreement in terms of the changes in the transmission spectra as the surrounding refractive index increases, and this can be seen in the
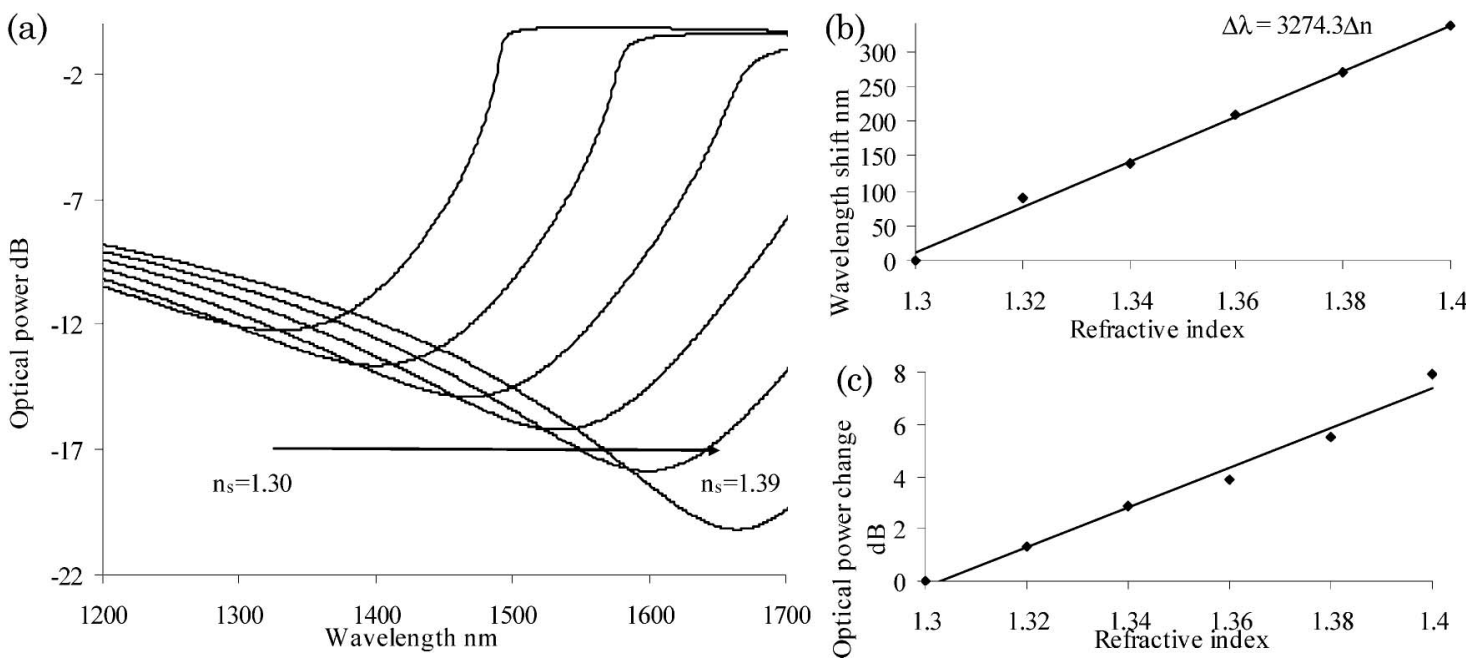

Fig. 12. Predicted spectral behavior of a Ge-coated D-shaped fiber as a function of the surrounding medium's index: (a) spectral variations, (b) wavelength shift, and (c) coupling strength as a function of index. 

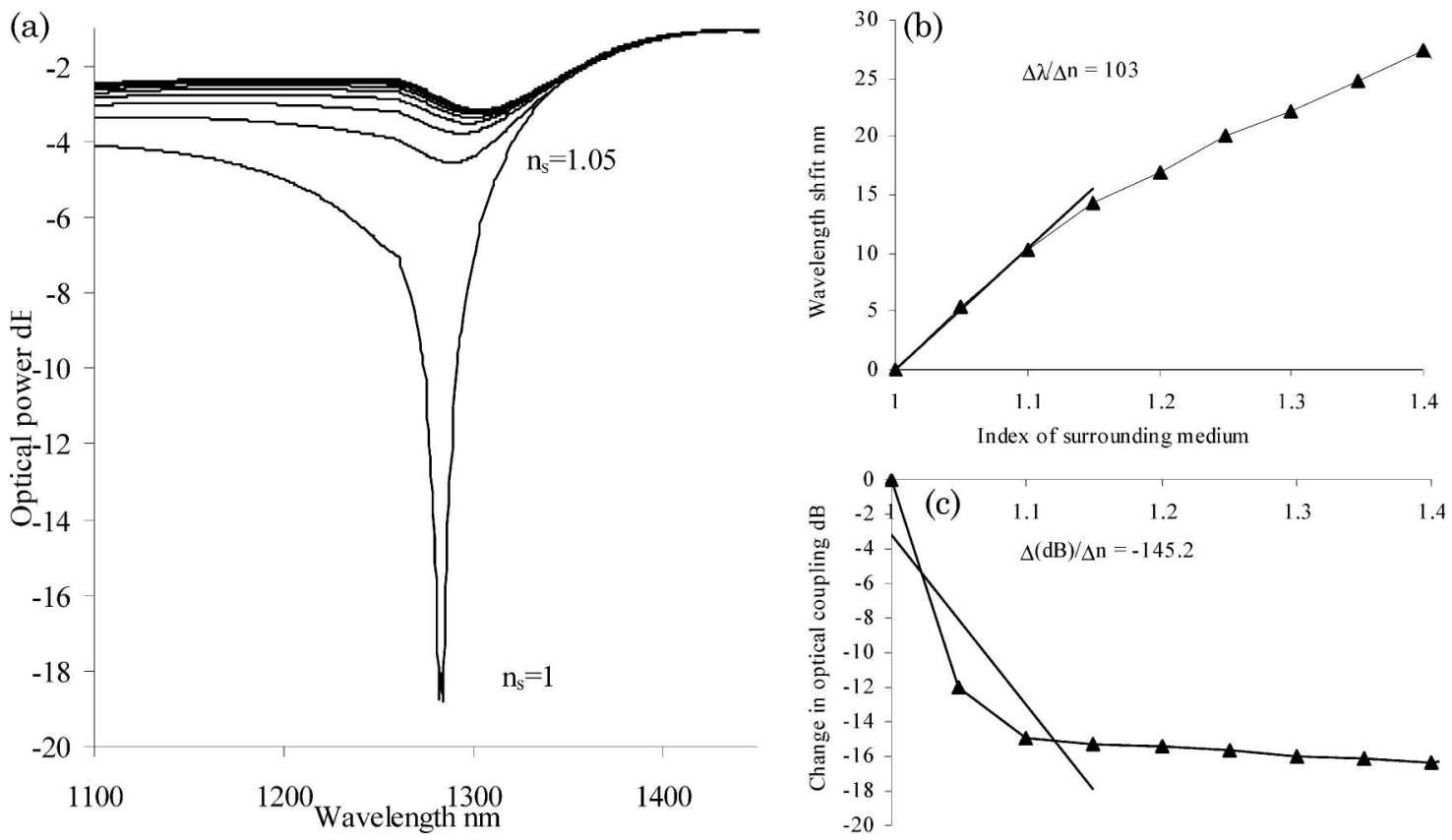

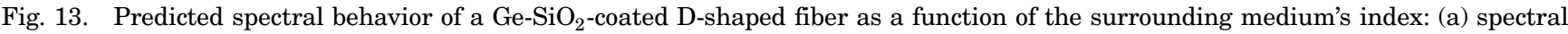
variations, (b) wavelength shift, and (c) coupling strength as a function of index with a thickness of $49.8 \mathrm{~nm}$ of germanium (roughness $\sim 12 \mathrm{~nm}$, thickness ranging up to $80 \mathrm{~nm}$,) and $30.2 \mathrm{~nm}$ of silica (roughness $\sim 9 \mathrm{~nm}$, thickness ranging up to $58 \mathrm{~nm}$,) using a fractional $V_{r}$ of 0.20 .

variations in the coupling strength of the SP. It should be noted that the parameters used in the model to obtain results similar to the experimental data differ from those used in actual the fabrication of the fiber device These differences may be due to experimental error in the calibration procedure for determining the thicknesses of the materials used along with the aforementioned inhomogeneous energy deposition, which changes the physical properties of the germanium $[16,17]$. What is consistent between theory and experimental observations is the large changes in coupling from air to an index 1.3. This may be expected considering that to support a SP the permittivity of one of the materials needs to be negative. In the case of germanium, the condition of negative permittivity exists in several locations in the spectrum with one of these windows ranging from $1250 \mathrm{~nm}$ to $1520 \mathrm{~nm}$, [24]. Therefore as the surrounding medium's refractive index increases the resonant condition for coupling shifts to longer wavelengths where the permittivity becomes real and the SP cannot be supported, thus resulting in large changes in coupling with small changes in refractive index. In the predicted spectral response we do not see the resonance coupling in the spectral range $1520 \mathrm{~nm}$ to $1630 \mathrm{~nm}$ due to the positive permittivity, but experimentally we do observe coupling; see Fig. 10, where resonance is observed at $1590 \mathrm{~nm}$ and
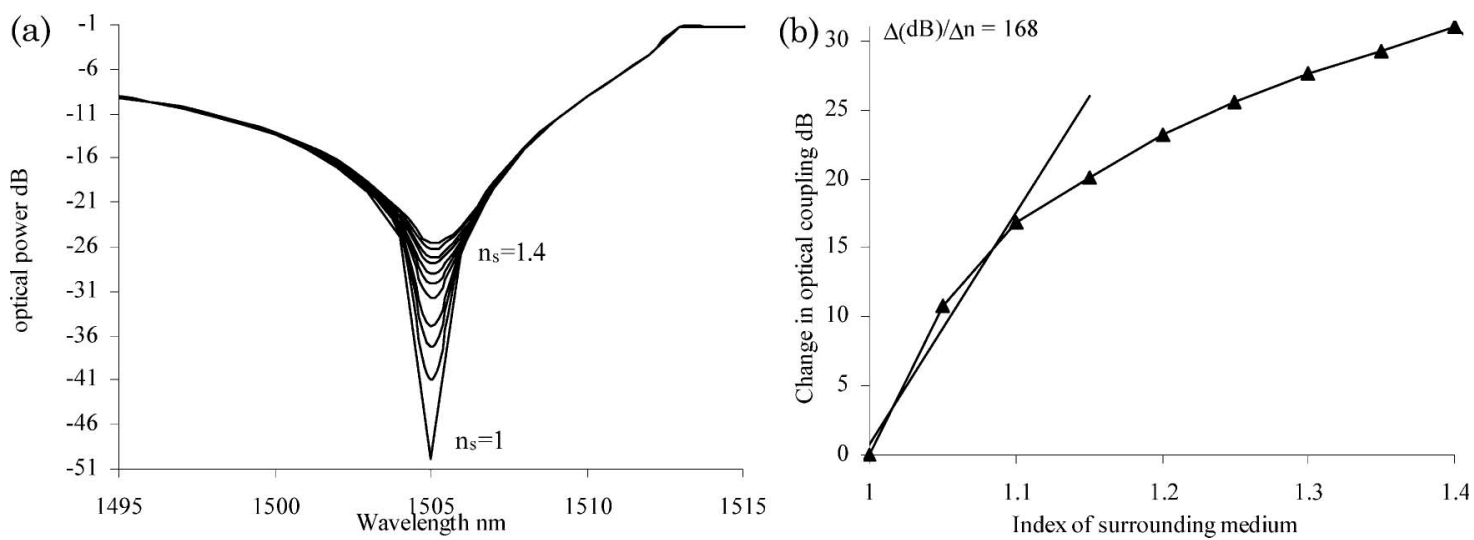

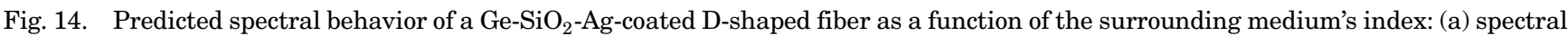
variations, (b) wavelength shift, and (c) coupling strength as a function of index with a thickness of $49.8 \mathrm{~nm}$ of germanium (roughness $12 \mathrm{~nm}$, thickness ranging up to $80 \mathrm{~nm}$ ), $30.2 \mathrm{~nm}$ of silica (roughness $9 \mathrm{~nm}$, thickness ranging up to $58 \mathrm{~nm}$ ), using a fractional volume $V_{r}$ of 0.50 and $38 \mathrm{~nm}$ of silver (roughness $5 \mathrm{~nm}$ with the thickness ranging up to $68 \mathrm{~nm}$ ) using a fractional $V_{r}$ of 0.60 . 
longer wavelengths, suggesting again UV-induced changes to the optical properties of germanium, consistent with $[16,17]$.

To attach any worth to these fiber sensors, their performance has to be compared to other sensor types in terms of wavelength shift (spectral sensitivity) and coupling strength variation (intensity sensitivity) as a function of index change. Using these predictions we estimate the spectral sensitivity of the $\mathrm{Ge}_{-} \mathrm{SiO}_{2}$ fiber device for low refractive indices from 1 to 1.1 to be $\Delta \lambda / \Delta n \sim 104 \mathrm{~nm}$, leading to an index resolution of $\sim 9 \times 10^{-4}$ (again assuming a spectral interrogation technique with a resolution of $0.1 \mathrm{~nm}$ ). Considering the optical power variation of the $\mathrm{Ge}_{-} \mathrm{SiO}_{2}$-coated device, a limiting index resolution of $\sim 6 \times 10^{-4}$ is possible (assuming $0.1 \mathrm{~dB}$ detection resolution). Considering the optical power variation of the $\mathrm{Ge}_{-} \mathrm{SiO}_{2}$-Ag-coated device, a limiting index resolution of $\sim 4 \times 10^{-4}$ is possible (assuming $0.1 \mathrm{~dB}$ detection resolution), this estimate is obtained from the model described above, which yields an optical power sensitivity $(\Delta I / \Delta n)$ of $165 \mathrm{~dB}$.

The sensitivities of these two devices are comparable to other fiber-based index sensors in the aqueous index regime, but at the low index regime (1 to 1.1) these devices' estimated sensitivities are increased fourfold $(\Delta \lambda / \Delta n 17 \mathrm{~nm})$ over, for example, LPGs, which are based upon the interaction of the evanescent electric field of the cladding modes with the surrounding medium [11,12]. In Fig. 15, a spectral sensitivity comparison is given between our coated devices and LPGs in a single mode fiber, based upon the combination of predicted theoretical results and experimental data to show the potential of these devices. The dramatic improvement in sensitivity/ performance over LPG fiber devices can be seen in Fig. 16. Inspecting Figs. 15 and 16 shows that the LPG is significantly less sensitive than the germanium-coated and the $\mathrm{Ge}_{-} \mathrm{SiO}_{2}$-Ag-coated devices in the aqueous index regime and also is outperformed by the $\mathrm{Ge}_{-} \mathrm{SiO}_{2}$-coated and the $\mathrm{Ge}-\mathrm{SiO}_{2}$-Ag-coated devices in the low index regime, thus our devices potentially yield higher index resolution than LPGs in both index regimes.

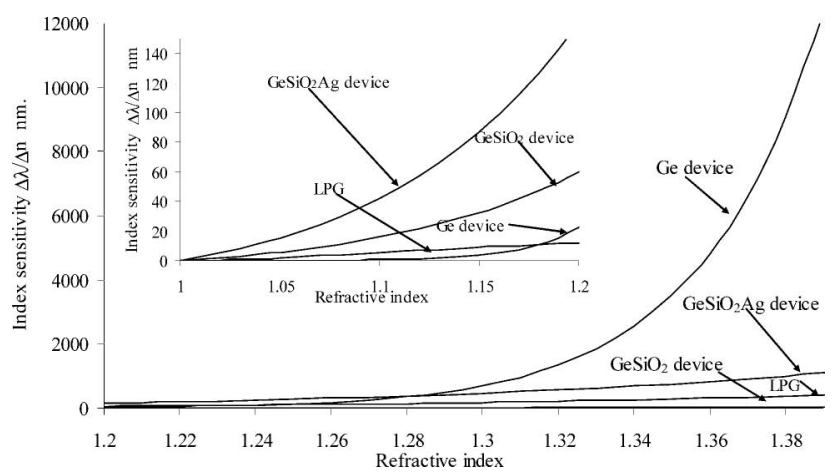

Fig. 15. Wavelength spectral sensitivity comparison of the three kinds of coated fiber devices as a function of refractive index along with a $L P G($ period $=240 \mu \mathrm{m}$, length $5 \mathrm{~cm})$.

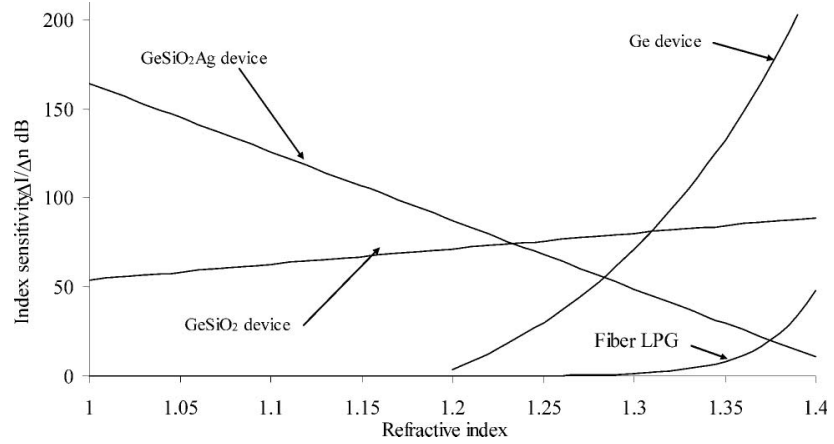

Fig. 16. Optical power sensitivity comparison of the three kinds of coated fiber devices as a function of refractive index along with a LPG $($ period $=240 \mu \mathrm{m}$, length $5 \mathrm{~cm})$.

\section{Conclusion}

We have investigated several surface plasmon resonance fiber (SPR) devices based upon single-layered and multilayered thin films deposited on the flat side of a lapped D-shaped fiber with UV exposure through a conventional fiber phase mask. The various devices yielded different index sensitivities, with the single Ge-coated device possessing the highest sensitivity in the aqueous regime, while evidence suggests that the $\mathrm{Ge}-\mathrm{SiO}_{2}-\mathrm{Ag}$-coated device should be the most sensitive at low indices, both devices outperforming LPGs in these regimes. Some of the SPR devices have high coupling efficiency in excess of $40 \mathrm{~dB}$ in air and the $\mathrm{Ge}_{-} \mathrm{SiO}_{2}$-Ag-coated device possessed an estimated index spectral sensitivity of $\Delta \lambda / \Delta n=90 \mathrm{~nm}$ and a coupling strength sensitivity of $\Delta I / \Delta n=$ $165 \mathrm{~dB}$ in the index range from 1 to 1.15 . A device with a single layer of Ge demonstrated an index spectral sensitivity of $\Delta \lambda / \Delta n=6790 \mathrm{~nm}$ over the index range of 1.33 to 1.36 with higher sensitivities obtained at higher indices.

\section{References}

1. S. Vasilev and O. Medvedkov, "Long-period refractive index fiber gratings: properties, applications and fabrication techniques," Proc. SPIE 4083, 212-223 (2000).

2. K. Schroeder, W. Ecke, R. Mueller, R. Willsch, and A. Andreev, "A fiber Bragg grating refractometer," Meas. Sci. Technol. 12, 757-764 (2001)

3. M. Piliarik, J. Homola, Z. Maníková, and J. Ctyroký, "Surface plasmon resonance sensor based on a single-mode polarization-maintaining optical fiber," Sens. Actuators B 90, 236-242 (2003).

4. J. Homola, S. Yee, and G. Gauglitz, "Surface plasmon resonance sensors: review," Sens.Actuators B 54, 3-15 (1999).

5. T. Erdogan, "Fiber grating spectra," J. Lightwave Technol. 15, 1277-1292 (1997).

6. S. Patskovsky, A. Kabashin, M. Meunier, and J. Luong, "Properties and sensing characteristics of surface plasmon resonance in infrared light," J. Opt. Soc. Am. A 20, 1644-1650 (2003).

7. H. Raether, ed.,Surface Plasmons on smooth and Rough Surfaces and on Gratings (Academic, 1997).

8. J. M. Brockman, B. P. Nelson, and R. M. Corn, "Surface plasmon resonance imaging measurement of ultra-thin organic films," Annu. Rev. Phys. Chem. 51, 41-63 (2000). 
9. M. Iga, A. Seki, and K. Watanabe, "Gold thickness dependence of SPR-based hetero-core structured optical fiber sensor," Sens. Actuators B 106, 363-368 (2005).

10. T. Allsop, R. Neal, S. Rehman, D. J. Webb, D. Mapps, and I. Bennion, "Surface plasmon resonance generation utilising gratings for biochemical sensing," in Optical Fiber Sensors (OFS) (Optical Society of America, 2006), paper WA4.

11. T. Allsop, R. Neal, S. Rehman, D. J. Webb, D. Mapps, and I. Bennion, "Characterization of infrared surface plasmon resonances generated from fiber optical sensor utilizing tilted Bragg gratings," J. Opt. Soc. Am. B 25, 481-490 (2008).

12. A. K. Sharma, Rajan, and B. D. Gupta, "Influence of dopants on the performance of a fiber optic surface plasmon resonance sensor," Opt. Commun. 274, 320-326 (2007).

13. M. Csete, A. Kőházi-Kis, Cs. Vass, Á. Sipos, G. Szekeres, M. Deli, K. Osvay, and Zs. Bor, "Atomic force microscopical and surface plasmon resonance spectroscopical investigation of sub-micrometer metal gratings generated by UV laserbased two-beam interference in Au-Ag bimetallic layers," Appl. Surf. Sci. 253, 7662-7671 (2007).

14. D. L. Williams, S. T. Davey, R. Kashyap, J. R. Armitage, and B. J. Ainslie, "UV spectroscopy of optical fibers and preforms," Proc. SPIE 1516, 29-37 (1991).

15. T. Allsop, R. Neal, P. Brown, S. Saied, S. Rehman, K. Kalli, D. J. Webb, J. Sullivan, D. Mapps, and I. Bennion, "A surface plasmon resonance fiber device for environmental sensing," Proc. SPIE 7004, 700441 (2008)

16. J. F. Young, J. E. Sipe, and H. M. van Driel, "Regimes of laserinduced periodic surface structure on germanium: radiation remnants and surface plasmons," Opt Lett. 8, 431-433 (1983).

17. J. F. Young, J. F. Young, J. E. Sipe, and H. M. van Driel, "Laserinduced periodic surface structure. III. Fluence regimes, the role of feedback, and details of the induced topography in germanium," Rev. B 30, 2001-2015 (1984).
18. E. M. Yeatman, "Resolution and sensitivity in surface plasmon microscopy and sensing," Biosensors Bioelect. 11, 635-649 (1996).

19. T. Allsop, R. Neal, S. Rehman, D. J. Webb, D. Mapps, and I. Bennion, Appl. Opt. 46, 5456-5470 (2007).

20. T. Allsop, D. J. Webb, and I. Bennion, "A comparison of the sensing characteristics of long period gratings written in three different types of fiber," Opt. Fiber Technol. 9, 210-223 (2003).

21. T. Allsop, R. Reeves, D. J. Webb, I. Bennion, and R. Neal, "A high sensitivity refractometer based upon a long period grating Mach-Zehnder interferometer," Rev. Sci. Instrum. 73, 1702-1705 (2002).

22. T. Allsop, F. Floreani, K. Jedrzejewski, P. Marques, R. Romero, D. Webb, and I. Bennion, "Refractive index sensing with longperiod grating fabricated in biconical tapered fibre," Electron. Lett. 41, 471-472 (2005).

23. R. Slavík, J. Homola, and J. Čtryoký, "Single mode optical fiber plasmon resonance sensor," Sens. Actuators B 54, 74-79 (1999).

24. E. Palik and G. Ghosh, Handbook of Optical Constants (Academic, 1998).

25. NanoRule+, http://www.pacificnanotech.com/.

26. D. E. Aspnes, J. B. Theeten, and F. Hottier, "Investigation of effective-medium models of microscopic surface roughness by spectroscopic ellipsometry," Phys. Rev. B 20, 3292-3302 (1979).

27. K. Johansen, H. Arwin, I. Lundström, and B. Liedberg, "Imaging surface plasmon resonance sensor based on multiple wavelengths: Sensitivity considerations," Rev. Sci. Instrum. 71, 3530-3538 (2000).

28. W. Brattain and H. Briggs, "The optical constants of germanium in the infra-red and visible," Phys. Rev. 75, 1705-1710 (1949). 